TRANSACTIONS OF THE

AMERICAN MATHEMATICAL SOCIETY

Volume 353, Number 2, Pages 427-440

S 0002-9947(00)02736-7

Article electronically published on October 23, 2000

\title{
SOME RESULTS ON INCREMENTS OF THE PARTIALLY OBSERVED EMPIRICAL PROCESS
}

\author{
ZACHARIE DINDAR
}

\begin{abstract}
The author investigates the almost sure behaviour of the increments of the partially observed, uniform empirical process. Some functional laws of the iterated logarithm are obtained for this process. As an application, new laws of the iterated logarithm are established for kernel density estimators.
\end{abstract}

\section{INTRODUCTION AND STATEMENT OF MAIN RESULT}

Let $\alpha_{n}(t)=n^{1 / 2}\left(F_{n}(t)-t\right)$ for $0 \leq t \leq 1$ denote the empirical uniform process, where $F_{n}(t)=n^{-1} \#\left\{U_{i} \leq t: 1 \leq i \leq n\right\}$ is the uniform empirical distribution function, based on the first $n \geq 1$ observations from a sequence $U_{1}, U_{2}, \ldots$ of independent uniform $(0,1)$ random variables, defined on a probability space $(\Omega, \mathcal{A}, \mathbb{P})$. Here \#A denotes the cardinality of $A$.

For any $0 \leq a<b \leq 1$, define the set $T=T_{N}=\left\{t_{1}, \ldots, t_{N}\right\} \subseteq[0,1]$, with $a<t_{1}=t_{1, N}<\ldots<t_{N}=t_{N, N}<b$ and $N=N_{n} \geq 1$.

In this paper, we investigate the almost sure behaviour of the increments of the partially observed empirical process $\left\{\alpha_{n}(t): t \in T\right\}$. For the statement of these results, the following notation is needed. For any $0 \leq h \leq 1$ and $n \geq 1$, define the empirical increments

$$
\xi_{n}(h, t ; s)=\alpha_{n}(t+s h)-\alpha_{n}(t), \quad t \in[0,1-h], s \in[0,1] .
$$

We refer to Shorack and Wellner [21] for the basic properties of the empirical process and Deheuvels [4], Deheuvels and Révész [9], Deheuvels and Mason [5, 6, 8] among others, for a description of the behaviour of the empirical increment process.

In what follows, $B[0,1]$ denotes the set of all bounded functions on $[0,1]$ and $A C[0,1]$, the set of all absolutely continuous functions $f \in B[0,1]$ of the form $f(t)=\int_{0}^{t} \dot{f}(s) d s$ for any $t \in[0,1]$ with $\int_{0}^{1} \dot{f}^{2}(s) d s<\infty$. We endow $B[0,1]$ with the topology defined by the sup-norm $\|f\|=\sup _{0 \leq s \leq 1}|f(s)|$.

For any $c \geq 0$, denote by $\mathcal{S}_{c}$ the set of all functions of $A C[0,1]$ satisfying $\int_{0}^{1} \dot{f}^{2}(s) d s \leq c^{2}$ and $f(0)=0$. Note that $\mathcal{S}_{c}=c \mathcal{S}_{1}$, where $\mathcal{S}:=\mathcal{S}_{1}$ is the Strassen set (see e.g. Strassen [22]). An application of the Arzela-Ascoli theorem shows that $\mathcal{S}_{c}$ is a compact subset of $(B[0,1],\|\cdot\|)$.

For any $A \subseteq B[0,1], B \subseteq B[0,1], \varepsilon>0, f \in B[0,1]$ and $g \in B[0,1]$, we set $d(f, B)=\inf _{g \in B[0,1]}\|f-g\|$ and $\delta(A, B)=\sup _{f \in A} d(f, B)$. The Hausdorff distance between $A$ and $B$ [endowed with the topology of the sup-norm], is defined

Received by the editors March 26, 1999 and, in revised form, April 26, 2000.

2000 Mathematics Subject Classification. Primary 60F17; Secondary 62G07.

Key words and phrases. Empirical processes, law of the iterated logarithm, functional laws.

(C)2000 American Mathematical Society 
by $\Delta(A, B)=\delta(A, B) \wedge \delta(B, A)=\inf \left\{\varepsilon>0: A \subseteq B^{\varepsilon}\right.$ and $\left.B \subseteq A^{\varepsilon}\right\}$. Here and elsewhere, $A^{\varepsilon}=\bigcup_{f \in A} N_{\varepsilon}(f)$ with $N_{\varepsilon}(f)=\{g \in B[0,1]:\|f-g\|<\varepsilon\}$.

Let $\left\{a_{n}: n \geq 1\right\}$ be a sequence of constants satisfying

(A1) $a_{n} \downarrow 0, n a_{n} \uparrow \infty$ and $0<a_{n}<1$,

(A2) $n a_{n} / \log n \rightarrow \infty$,

(A3) $\left(\log \left(1 / a_{n}\right)\right) /(\log \log n) \rightarrow \infty$.

Deheuvels and Mason [6] proved the following result.

Theorem A. Under (A1)-(A3), with probability 1,

$$
\lim _{n \rightarrow \infty} \Delta\left(\left\{\left(2 a_{n} \log \left(1 / a_{n}\right)\right)^{-1 / 2} \xi_{n}\left(a_{n}, t ; .\right): 0 \leq t \leq 1-a_{n}\right\}, \mathcal{S}\right)=0 .
$$

Our aim is to obtain the analog of Theorem $\mathrm{A}$ when $\left\{\alpha_{n}(t): 0 \leq t \leq 1\right\}$ is replaced by the partially observed empirical process. Towards this goal, a methodology exposed by Weber [26], Weber [27, Lifshits and Weber [17] is used. We refer to these articles for the study of limit laws for the partially observed Wiener process.

Let $\left\{h_{n}: n \geq 1\right\}$ denote a sequence of constants such that

(H1) $h_{n} \downarrow 0, n h_{n} \uparrow \infty$, and $0<h_{n}<1$,

(H2) $n h_{n} / \log n \rightarrow \infty$,

(H3) $\log N_{n} / \log \log n \rightarrow \infty$,

(H4) $\log N_{n} / \log \left(1 / h_{n}\right) \rightarrow d \in[0, \infty]$.

Remark 1.1. Here and elsewhere, we suppose the existence of a sequence of positive constants $\left\{\ell_{n}: n \geq 1\right\}$ such that $N_{n}=\left\lfloor\ell_{n}\right\rfloor$ for any $n \geq 1$ and $\ell_{n} \uparrow \infty, n^{-1} \ell_{n} \downarrow 0$ as $n \rightarrow \infty$. Here $\lfloor u\rfloor \leq u<\lfloor u\rfloor+1$ denotes the integer part of $u$.

For any $n \geq 1$, set

$$
c_{n, N}=c_{n, N_{n}}=\left\{2 h_{n}\left(\log N_{n}+\log _{2} n\right)\right\}^{1 / 2},
$$

where $\log _{+} u=\log (u \vee e)$ and $\log _{2} u=\log \left(\log _{+} u\right)$ for $u \in \mathbb{R}$. The purpose of this paper is to establish the following theorem.

Theorem 1.1. (i) Under (H1), (H2), (H3), (H4) with $d \in[0,1)$, we have with probability 1 ,

$$
\lim _{n \rightarrow \infty} \Delta\left(\left\{c_{n, N}^{-1} \xi_{n}\left(h_{n}, t ; .\right): t \in T_{n}\right\}, \mathcal{S}\right)=0 .
$$

(ii) Under (H1), (H2), (H3), (H4) with $d \in[1, \infty]$, we have with probability 1,

$$
\lim _{n \rightarrow \infty} \Delta\left(\left\{\left(2 h_{n} \log \left(1 / h_{n}\right)\right)^{-1 / 2} \xi_{n}\left(h_{n}, t ; .\right): t \in T_{n}\right\}, \mathcal{S}\right)=0 .
$$

Remark 1.2. A natural question concerns the validity of Theorem 1.1 when the technical condition (H3) is no longer satisfied. In Dindar [1], we prove that under (H1), (H2) and assumption $\log N_{n} / \log \log n \rightarrow c \in[0, \infty)$ as $n \rightarrow \infty$, we have with probability 1 ,

$$
\begin{gathered}
\lim _{n \rightarrow \infty} \delta\left(\mathcal{S}_{c /(c+1)},\left\{c_{n, N}^{-1} \xi_{n}\left(a_{n}, t ; .\right): t \in T_{n}\right\}\right)=0, \quad \text { and } \\
\lim _{n \rightarrow \infty} \delta\left(\left\{c_{n, N}^{-1} \xi_{n}\left(a_{n}, t ; .\right): t \in T_{n}\right\}, \mathcal{S}\right)=0 .
\end{gathered}
$$

We refer to Dindar [11] for further comments and a complete proof of these last results. 


\section{Proofs}

We postpone the proof of statements (1.4) and (1.5) until Sections 2.2 and 2.3. Below, we introduce some facts and notations suitable for our needs.

\subsection{Facts and notations.}

Remark 2.1. Under assumption (H3) of Theorem 1.1, it is easy to see that the sequence of norming constants $c_{n, N}$ can be replaced by $d_{n, N}=\left(2 h_{n} \log N_{n}\right)^{1 / 2}$ for any $n \geq 1$.

Let $\{W(t): 0 \leq t \leq 1\}$ denote a standard Wiener process and let $\left\{\Pi_{n}(t): t \geq 0\right\}$ denote a sequence of right continuous Poisson processes such that $\mathbb{E}\left(\Pi_{n}(t)\right)=n t$ for all $n \geq 1$ and $t \geq 0$. For any $h \in(0,1), s \in[0,1], t \geq 0$ and integers $n \geq 1$, set

$$
L_{n}(h, t ; s)=n^{-1 / 2}\left\{\Pi_{n}(t+s h)-\Pi_{n}(t)-n s h\right\} .
$$

Fact 1. For all $n$ sufficiently large and $1 \leq m \leq h_{n}^{-1}$, for any $t_{i} \in[0,1)$ such that $t_{i+1}-t_{i} \geq h_{n}$ with $i=0,1, \ldots, m-1$ and Borel sets $B_{1}, \ldots, B_{m} \in(B[0,1],\|\cdot\|)$,

$$
\begin{aligned}
& \mathbb{P}\left(\xi_{n}\left(h_{n}, t_{i} ; .\right) \notin B_{i}, i=0, \ldots, m-1\right) \\
& \quad \leq 2 \mathbb{P}\left(L_{n}\left(h_{n}, t_{i} ; .\right) \notin B_{i}, i=0, \ldots, m-1\right) .
\end{aligned}
$$

Proof. See e.g. Deheuvels and Mason [6].

Let $\{\Pi(t): t \geq 0\}$ be a standard Poisson process. We will make use of the strong approximation results of Komlós, Major and Tusnády [14, 15, 16] (see e.g. Deheuvels and Mason [7, p. 85]) to construct on the same probability space a version of the process $\{\Pi(t): t \geq 0\}$ and a standard Wiener process $\{W(t): t \geq 0\}$, such that

Fact 2. For all $T \geq 1$ and $z \in \mathbb{R}$,

$$
\mathbb{P}\left(\sup _{0 \leq x \leq T}|\Pi(x)-x-W(x)| \geq C_{1} \log T+z\right) \leq C_{2} \exp \left(-C_{3} z\right),
$$

for some universal constants $C_{1}, C_{2}$ and $C_{3}$.

For any $f \in B[0,1]$, set

$$
J(f)= \begin{cases}\int_{0}^{1}(\dot{f}(s))^{2} d s & \text { if } f \in A C[0,1] \\ \infty & \text { otherwise. }\end{cases}
$$

For any $B \subseteq B[0,1]$, set

$$
J(B)=\inf _{f \in B} J(f) .
$$

Note that $J: B[0,1] \longrightarrow[0, \infty]$ is a lower semi-continuous function. For further properties, we refer to Varadhan [25, Deuschel and Stroock [10. p. 12].

For any $\lambda>0$ and $0 \leq t \leq 1$, set

$$
W_{\lambda}(t)=2^{-1 / 2} \lambda^{-1} W(\lambda t) .
$$

We will make use of the following result stated below.

Fact 3. (i) For any closed subset $F$ of $B[0,1]$ endowed with the sup-norm topology,

$$
\limsup _{\lambda \rightarrow \infty} \lambda^{-1} \log \mathbb{P}\left(W_{\lambda} \in F\right) \leq-J(F) .
$$


(ii) For any open subset $G$ of $B[0,1]$ endowed with the sup-norm topology,

$$
\limsup _{\lambda \rightarrow \infty} \lambda^{-1} \log \mathbb{P}\left(W_{\lambda} \in G\right) \geq-J(G) .
$$

Proof. See e.g. Schilder [20] or Deuschel and Stroock [10, Theorem 1.3.27].

Introduce the Chernoff function of a standard Poisson process (see e.g. Chernoff [2] or Shorack and Wellner [21, pp. 432-433 and 856]) defined by

$$
h(r)= \begin{cases}r \log r-r+1 & \text { for } r>0 \\ 1 & \text { if } r=0 \\ \infty & \text { otherwise }\end{cases}
$$

Introduce the function

$$
\Psi(x)= \begin{cases}2 h(1+x) / x^{2} & \text { for } x \geq-1 \text { and } x \neq 0 \\ 1 & \text { if } x=0 \\ \infty & \text { otherwise }\end{cases}
$$

Remark 2.2. We refer to Shorack and Wellner [21, pp. 440-445] for a detailed study of $\Psi$ properties. Among others, note that $\Psi$ is a non-increasing function, and that $\Psi(x) \uparrow 1$ when $x \downarrow 0$.

We will need the following inequalities.

Fact 4. For any $0<a \leq 1 / 2, \lambda>0$ and integers $n \geq 1$,

$$
\mathbb{P}\left(\left\|\frac{\alpha_{n}}{1-I}\right\|_{0}^{a} \geq \frac{\lambda}{1-a}\right) \leq \exp \left(-\frac{\lambda^{2}}{2 a(1-a)} \Psi\left(\frac{\lambda}{a \sqrt{n}}\right)\right) .
$$

Proof. See e.g. James [13] or Shorack and Wellner [21, p. 444].

Fact 5. For any $0<a \leq 1 / 2, \lambda>0$ and integers $n \geq 1$,

$$
\mathbb{P}\left(\sup _{0 \leq s \leq a} n\left|F_{n}(s)-s\right| \geq \lambda\right) \leq 2 n a / \lambda^{2} .
$$

Proof. Remark that $\left\{n\left(F_{n}(s)-s\right) /(1-s): 0 \leq s<1\right\}$ is a martingale (see e.g. Shorack and Wellner [21, p. 133]). Therefore,

$$
\mathbb{P}\left(\sup _{0 \leq s \leq a} n\left|F_{n}(s)-s\right| \geq \lambda\right) \leq \frac{\mathbb{E}\left(n^{2}\left|F_{n}(a)-a\right|^{2}\right)}{(\lambda(1-a))^{2}} \leq \frac{2 n a}{\lambda^{2}},
$$

which concludes the proof.

\subsection{Proof of Theorem 1.1 (i).}

2.2.1. Preliminary lemmas. For any integers $Q \geq 1$, let

$$
d_{n, Q}:=\left(2 h_{n} \log _{+} Q\right)^{1 / 2} .
$$

For any $0<h<1 / 2$ and $n \geq 1$, define

$$
\omega_{n, N}(h)=\max _{i=1, \ldots, N} \sup _{0 \leq s \leq 1}\left|\alpha_{n}\left(t_{i}+s h\right)-\alpha_{n}\left(t_{i}\right)\right|,
$$

where $t_{i} \in T$ for $1 \leq i \leq N$. We have the following lemma.

Lemma 2.1. For any $0<h<1 / 2$ and $n \geq 1$,

$$
\mathbb{P}\left(\omega_{n, N}(h) \geq \lambda h^{1 / 2}\right) \leq N \exp \left(-\frac{\lambda^{2}}{2}(1-h) \Psi\left(\frac{\lambda}{\sqrt{n h}}\right)\right) .
$$


Proof. Statement (2.15) follows from (2.14), Fact 4 with $\lambda$ replaced by $\lambda \sqrt{h}(1-h)$, $a=h$, and the fact that $\Psi$ is non-increasing.

Let $\theta>0$ whose value will be set elsewhere. For any $k \geq 0$, define

$$
\mu_{k}=\left\lfloor(1+\theta)^{k}\right\rfloor \text {. }
$$

We record the following result for later use.

Lemma 2.2. Under (H1), (H2), (H3), for any $\varepsilon>0$, there exists $\theta:=\theta(\varepsilon)>0$, with $k(\varepsilon)<\infty$, such that for any $k \geq k(\varepsilon)$

$$
\begin{aligned}
& \mathbb{P}\left(\max _{\mu_{k-1}<n \leq \mu_{k}} d_{n, N_{n}}^{-1} \omega_{n, N_{n}}\left(h_{n}\right) \geq 1+2 \varepsilon\right) \\
& \quad \leq 2 \mathbb{P}\left(\frac{\omega_{\mu_{k+1}, N_{\mu_{k+1}}}\left((1+\theta)^{2} h_{\mu_{k}}\right)}{(1+\theta) \sqrt{h_{\mu_{k}}}} \geq \frac{1+\varepsilon}{1+\theta}\left(2 \log N_{\mu_{k-1}}\right)^{1 / 2}\right) .
\end{aligned}
$$

Proof. This lemma is inspired by a result of Stute [23]. The proof is omitted for the sake of conciseness. We refer to Shorack and Wellner [21, pp. 545, 550-552] for a glimpse of the demonstration.

We will need the following lemma.

Lemma 2.3. Under (H1), (H2), (H3), with probability 1,

$$
\limsup _{n \rightarrow \infty} c_{n, N}^{-1} \omega_{n, N}\left(h_{n}\right) \leq 1
$$

Proof. Fix $\varepsilon>0$ and consider the event

$$
E_{n}=\left\{\frac{\omega_{n, N_{n}}\left(h_{n}\right)}{\sqrt{h_{n}}} \geq(1+\varepsilon)\left(2 \log N_{n}\right)^{1 / 2}\right\} .
$$

Assertion (2.16) is verified as soon as $\sum_{n} \mathbb{P}\left(E_{n}\right)<\infty$. Hence using Lemma 2.2 and the Borel-Cantelli lemma, it suffices to show that $\sum_{k} \mathbb{P}\left(F_{k}\right)<\infty$, where

$$
F_{k}=\left\{\frac{\omega_{\mu_{k+1}, N_{\mu_{k+1}}}\left((1+\theta)^{2} h_{\mu_{k}}\right)}{(1+\theta) \sqrt{h_{\mu_{k}}}} \geq \frac{1+\varepsilon}{1+\theta}\left(2 \log N_{\mu_{k-1}}\right)^{1 / 2}\right\} .
$$

For any $m \geq 1$, let $\lambda_{m}=\left(2 \log N_{m}\right)^{1 / 2}$. We use Lemma 2.1 with $\lambda$ replaced by $(1+\varepsilon)(1+\theta)^{-1} \lambda_{\mu_{k-1}}$ and $h=(1+\theta) \sqrt{h_{\mu_{k}}}$ to obtain

$$
\begin{aligned}
\mathbb{P}\left(F_{k}\right) \leq N_{\mu_{k+1}} \exp \left\{-\frac{1}{2}\left(\frac{1+\varepsilon}{1+\theta}\right)^{2} \lambda_{\mu_{k-1}}^{2}\left(1-(1+\theta)^{2} h_{\mu_{k}}\right)\right. \\
\left.\times \Psi\left(\frac{1+\varepsilon}{(1+\theta)^{2}} \frac{\lambda_{\mu_{k-1}}}{\sqrt{\mu_{k+1} h_{\mu_{k}}}}\right)\right\} .
\end{aligned}
$$

Consider $\theta>0$ such that $\{(1+\varepsilon) /(1+\theta)\}^{2}\left(1-(1+\theta)^{2} h_{\mu_{k}}\right)>1+\varepsilon / 4$. This, combined with (2.17) entails

$$
\begin{aligned}
& \mathbb{P}\left(\frac{\omega_{\mu_{k+1}, N_{\mu_{k+1}}}\left((1+\theta)^{2} h_{\mu_{k}}\right)}{\sqrt{h_{\mu_{k}}}} \geq(1+\varepsilon) \lambda_{\mu_{k-1}}\right) \\
& \quad \leq N_{\mu_{k+1}} \exp \left\{-\frac{1}{2}\left(1+\frac{\varepsilon}{4}\right) \lambda_{\mu_{k-1}}^{2} \Psi\left(\frac{1+\varepsilon}{(1+\theta)^{2}} \frac{\lambda_{\mu_{k-1}}}{\sqrt{\mu_{k+1} h_{\mu_{k}}}}\right)\right\} .
\end{aligned}
$$

By (H1)-(H2) and Remark 1.2, we obtain for any $k$ sufficiently large

$$
\Psi\left(\frac{1+\varepsilon}{(1+\theta)^{2}} \frac{\lambda_{\mu_{k-1}}}{\sqrt{\mu_{k+1} h_{\mu_{k}}}}\right)>1-\varepsilon^{\prime},
$$


where $\varepsilon^{\prime}>0$ is such that $(1+\varepsilon / 4)\left(1-\varepsilon^{\prime}\right)>1+\varepsilon / 16$. This, combined with (2.19) implies

$$
\begin{aligned}
& \mathbb{P}\left(\frac{\omega_{\mu_{k+1}, N_{\mu_{k+1}}}\left((1+\theta)^{2} h_{\mu_{k}}\right)}{\sqrt{h_{\mu_{k}}}} \geq(1+\varepsilon) \lambda_{\mu_{k-1}}\right) \\
& \quad \leq N_{\mu_{k+1}} \exp \left\{-\left(1+\frac{\varepsilon}{16}\right) \log N_{\mu_{k-1}}\right\} .
\end{aligned}
$$

The definition of $\mu_{k}$ and a few computation prove that the RHS of (2.20) is bounded by $k^{-2}$ for any $k$ sufficiently large. Therefore, $\sum_{k} \mathbb{P}\left(F_{k}\right)<\infty$ and (2.16) is proved.

Let $\gamma>0$ be a constant whose value will be set elsewhere. For any $k \geq 1$, set

$$
n_{k}=\left\lfloor(1+\gamma)^{k}\right\rfloor \text {. }
$$

We have the following result.

Lemma 2.4. For any $k$ sufficiently large,

(1i) $\log n_{k} \sim k \log (1+\gamma)$,

(1ii) $\log _{2} n_{k} \sim \log k$,

(1iii) $\log _{2} n_{k} \sim \log _{2} n_{k+1}$,

(1iv) $n_{k+1}-n_{k}=(1+o(1)) \gamma n_{k}$.

Proof. Proofs of (1i)-(1iv) are trivial and therefore omitted.

For any $\varepsilon>0$ and $k \geq 1$, consider the sets

$$
\begin{aligned}
C_{k}(\varepsilon, \gamma)= & \left\{\left(n / n_{k+1}\right)^{1 / 2} d_{n_{k+1}, N_{n_{k+1}}}^{-1} \xi_{n}\left(h_{n_{k+1}}, t_{i} ; .\right) \notin \mathcal{S}^{\varepsilon},\right. \\
& \text { for } \left.i \in\left\{1, \ldots, N_{n_{k+1}}\right\} \text { and } n_{k}<n \leq n_{k+1}\right\}
\end{aligned}
$$

and $D_{k}(\varepsilon, \gamma)=\left\{d_{n_{k+1}, N_{n_{k+1}}}^{-1} \xi_{n_{k+1}}\left(h_{n_{k+1}}, t_{i} ;.\right) \notin \mathcal{S}^{\varepsilon}\right.$, for $\left.i \in\left\{1, \ldots, N_{n_{k+1}}\right\}\right\}$.

Lemma 2.5. For any $\varepsilon>0$ and $\gamma>0$, there exists a $k(\varepsilon, \gamma)<\infty$ such that, for any $k \geq k(\varepsilon, \gamma)$

$$
\mathbb{P}\left(C_{k}(\varepsilon, \gamma)\right) \leq 2 \mathbb{P}\left(D_{k}(\varepsilon / 2, \gamma)\right) .
$$

Proof. See e.g. Deheuvels and Mason [6]. Define the conditions

$$
\begin{gathered}
E_{k, i, n}(\varepsilon)=\left\{\left(\frac{n}{n_{k+1}}\right)^{1 / 2} d_{n_{k+1}, N_{n_{k+1}}}^{-1} \xi_{n}\left(h_{n_{k+1}}, t_{i} ; .\right) \notin \mathcal{S}^{\varepsilon}\right\}, \\
E_{k, n}=\bigcup_{i=1}^{N_{n_{k+1}}} E_{k, i, n}(\varepsilon), \\
F_{k, i, n}(\varepsilon)=\left\{d_{n_{k+1}, N_{n_{k+1}}}^{-1}\left\|\xi_{n_{k+1}}\left(h_{n_{k+1}}, t_{i} ; .\right)-\left(\frac{n}{n_{k+1}}\right)^{1 / 2} \xi_{n}\left(h_{n_{k+1}}, t_{i} ; .\right)\right\|<\varepsilon\right\} .
\end{gathered}
$$

For any $\varepsilon^{\prime}>0, \varepsilon^{\prime \prime}>0$, the conditions $\left\{E_{k, i, n}\left(\varepsilon^{\prime}\right): i \geq 1\right\}$ and $\left\{F_{k, i, n}\left(\varepsilon^{\prime \prime}\right): i \geq 1\right\}$ are independent. Therefore,

$$
\mathbb{P}\left(C_{k}(\varepsilon, \gamma)\right)=\sum_{q=n_{k}+1}^{n_{k+1}} \sum_{i=1}^{N_{n_{k+1}}} \mathbb{P}\left(E_{k, i, q}(\varepsilon) \cap_{j=1}^{i-1} \bar{E}_{k, j, q}(\varepsilon) \cap_{r=n_{k}+1}^{q-1} \bar{E}_{r, q}(\varepsilon)\right) .
$$


Here and elsewhere, $\bar{A}$ denotes the closure of the set $A$. Moreover, observe that

$$
\begin{aligned}
& \inf _{n_{k}<n \leq n_{k+1}} \inf _{1 \leq l \leq N_{n_{k+1}}} \mathbb{P}\left(F_{k, l, n}(\varepsilon / 2)\right) \mathbb{P}\left(C_{k}(\varepsilon, \gamma)\right) \\
& \leq \sum_{q=n_{k}+1}^{n_{k+1}} \sum_{i=1}^{N_{n_{k+1}}} \mathbb{P}\left(E_{k, i, q}(\varepsilon) \cap F_{k, i, q}(\varepsilon / 2) \cap_{j=1}^{i-1} \bar{E}_{k, j, q}(\varepsilon) \cap_{r=n_{k}+1}^{q-1} \bar{E}_{r, q}(\varepsilon)\right) \\
& \leq \mathbb{P}\left(\bigcup_{i=1}^{N_{n_{k+1}}} E_{k, i, n_{k+1}}(\varepsilon / 2)\right)=\mathbb{P}\left(D_{k}(\varepsilon / 2, \gamma)\right) .
\end{aligned}
$$

On the other hand, we have

$$
\mathbb{P}\left(\bar{F}_{k, l, n}(\varepsilon / 2)\right)=\mathbb{P}\left(n_{k+1}^{-1 / 2} \sup _{0 \leq u \leq h_{n_{k+1}}}\left(n_{k+1}-n\right)\left|F_{n_{k+1}-n}(u)-u\right| \geq \frac{\varepsilon}{2} d_{n_{k+1}, N_{n_{k+1}}}\right) .
$$

Next, use Fact 5 and (2.12) to obtain

$$
\mathbb{P}\left(\bar{F}_{k, l, n}(\varepsilon / 2)\right) \leq 8 \frac{\left(n_{k+1}-n_{k}\right) h_{n_{k+1}}}{\varepsilon^{2} n_{k+1} d_{n_{k+1}, N_{n_{k+1}}}^{2}} .
$$

Statement (1.3) and (H3) jointly imply that the RHS of (2.24) converges to 0 . Hence, $\left(\inf _{n_{k}<n \leq n_{k+1}} \inf _{1 \leq l \leq N_{n_{k+1}}} \mathbb{P}\left(F_{k, l, n}(\varepsilon / 2)\right)\right)^{-1} \rightarrow 1$ as $k \rightarrow \infty$. This, combined with (2.23), entails (2.22).

The blocking arguments described in the next two lemmas will be suitable for the proof of (1.4).

Lemma 2.6. Under (H1), (H2), (H3), with probability 1,

$$
\begin{aligned}
& \lim _{\gamma \downarrow 0} \limsup _{k \rightarrow \infty}\left\{\max _{n_{k}<n \leq n_{k+1}} \max _{1 \leq i \leq N_{n_{k+1}}}\right. \\
& \left.\left|\left(\frac{n}{n_{k+1}}\right)^{1 / 2} d_{n_{k+1}, N_{n_{k+1}}}^{-1}-d_{n, N_{n}}^{-1}\right| \times\left\|\xi_{n}\left(h_{n_{k+1}}, t_{i} ; .\right)\right\|\right\}=0 .
\end{aligned}
$$

Proof. Observe that

$$
\left|\left(\frac{n}{n_{k+1}}\right)^{1 / 2} d_{n_{k+1}, N_{n_{k+1}}}^{-1}-d_{n, N_{n}}^{-1}\right|=d_{n, N_{n}}^{-1}\left|\left(\frac{n}{n_{k+1}}\right)^{1 / 2} \frac{d_{n, N_{n}}}{d_{n_{k+1}, N_{n_{k+1}}}}-1\right| .
$$

By (H1), it is easy to see that $\sqrt{n} d_{n} \uparrow \infty$ as $n \rightarrow \infty$. Therefore, for any $k$ sufficiently large,

$$
\left|\left(\frac{n}{n_{k+1}}\right)^{1 / 2} \frac{d_{n, N_{n}}}{d_{n_{k+1}, N_{n_{k+1}}}}-1\right| \leq\left|\left(\frac{n_{k}}{n_{k+1}}\right)^{1 / 2} \frac{d_{n_{k}, N_{n_{k}}}}{d_{n_{k+1}, N_{n_{k+1}}}}-1\right| .
$$

Observe that (H1) and (2.21) imply for all $k$ large enough

$$
\frac{1}{1+\gamma} \leq \frac{n_{k} h_{n_{k}}}{n_{k+1} h_{n_{k+1}}} \leq 1
$$

Moreover, (H1), (H3) and (2.21) combined with Remark 1.1 entails for any $k$ large enough

$$
1-\frac{\log (1+\gamma)}{4 \log k} \leq \frac{\log N_{n_{k}}}{\log N_{n_{k+1}}} \leq 1 .
$$

Therefore, (2.28) and (2.29) jointly imply that the RHS of (2.26) converges to 0 as $k \rightarrow \infty$ and $\gamma \downarrow 0$. Next, we use (H1) and (2.14) to obtain for any $k$ large 
enough and $1 \leq i \leq N_{n_{k+1}}$, that $\left\|\xi_{n}\left(h_{n_{k+1}}, t_{i} ;.\right)\right\| \leq \omega_{n, N_{n_{k+1}}}\left(h_{n}\right)$. Moreover, for any $n_{k}<n \leq n_{k+1}$, we have $d_{n, N_{n_{k+1}}} d_{n, N_{n}}^{-1} \rightarrow 1$ as $k \rightarrow \infty$. These last statements, combined with Lemma 2.3 entail (2.25).

Lemma 2.7. Under (H1), (H2), (H3), we have with probability 1 ,

$$
\lim _{\gamma \downarrow 0} \limsup _{k \rightarrow \infty}\left\{\max _{n_{k}<n \leq n_{k+1}} \max _{1 \leq i \leq N_{n_{k+1}}} d_{n, N_{n}}^{-1}\left\|\xi_{n}\left(h_{n_{k+1}}, t_{i} ; .\right)-\xi_{n}\left(h_{n}, t_{i} ; .\right)\right\|\right\}=0 .
$$

Proof. For any $n_{k}<n \leq n_{k+1}$ and $1 \leq i \leq N_{n_{k+1}}$, we have

$$
\left\|\xi_{n}\left(h_{n_{k+1}}, t_{i} ; .\right)-\xi_{n}\left(h_{n}, t_{i} ; .\right)\right\| \leq \omega_{n, N_{n_{k+1}}}\left(h_{n}-h_{n_{k+1}}\right) .
$$

Moreover, for any $n_{k}<n \leq n_{k+1}$ and any $k$ large enough, (H1) implies

$$
h_{n}-h_{n_{k+1}} \leq h_{n}\left(1-\frac{h_{n_{k+1}}}{h_{n_{k}}}\right) \leq \gamma h_{n} .
$$

From Lemma 2.3, we get with probability 1

$$
\limsup _{k \rightarrow \infty} d_{n, N_{n_{k+1}}}^{-1} \omega_{n, N_{n_{k+1}}}\left(\gamma h_{n}\right) \leq \gamma^{1 / 2} .
$$

Next, observe that for any $n_{k}<n \leq n_{k+1}, d_{n, N_{n_{k+1}}} d_{n, N_{n}}^{-1} \longrightarrow 1$ as $k \rightarrow \infty$. This last statement, combined with (2.31)-(2.33) entails (2.30) as $k \downarrow 0$.

Proof of Theorem 1.1 (i) is divided in two steps.

2.2.2. Proof of Theorem 1.1 (i). First step. As a first step, we show that, for any $\varepsilon>0$, there exists with probability 1 an $n(\varepsilon)<\infty$ such that, for any $n \geq n(\varepsilon)$

$$
\mathcal{S} \subseteq\left\{d_{n, N}^{-1} \xi_{n}\left(h_{n}, t ; .\right): t \in T_{n}\right\}^{\varepsilon} .
$$

Recall that $\mathcal{S}$ is a compact subset of $(B[0,1],\|\|$.$) (cf. Conway [3, p. 177]). Hence,$ for any $\varepsilon>0$, we can choose an integer $m \geq 1$ and $f_{i} \in \mathcal{S}, 1 \leq i \leq m$, such that $\mathcal{S} \subseteq \bigcup_{i=1}^{m} N_{\varepsilon}\left(f_{i}\right)$. Therefore, $(2.34)$ will be verified as soon as we show that for any $f \in \mathcal{S}$ and $\varepsilon>0$, we have

$$
\sum_{n} P_{n}(f, \varepsilon):=\sum_{n} \mathbb{P}\left(\bigcap_{i=1}^{N_{n}} d_{n, N_{n}}^{-1} \xi_{n}\left(h_{n}, t_{i} ; .\right) \notin N_{\varepsilon}(f)\right)<\infty,
$$

where $t_{i} \in T$ for $i=1, \ldots, N_{n}$.

By Fact 1, it is easy to show that

$$
P_{n}(f, \varepsilon) \leq 2\left\{1-\mathbb{P}\left(\left(2 n h_{n} \log N_{n}\right)^{-1 / 2}\left(\Pi\left(n h_{n} .\right)-n h_{n} .\right) \in N_{\varepsilon}(f)\right)\right\}^{N_{n}} .
$$

Set $P_{n}^{(1)}(f, \varepsilon):=\mathbb{P}\left(\left\{2 n h_{n} \log N_{n}\right\}^{-1 / 2}\left\{\Pi\left(n h_{n}.\right)-n h_{n}.\right\} \in N_{\varepsilon}(f)\right)$ and let $\{W(t)$ : $t \geq 0\}$ denote a standard Wiener process such that (2.3) of Fact 2 is verified. For any $n \geq 1$, we have

$$
\begin{aligned}
P_{n}^{(1)}(f, \varepsilon) & \geq \mathbb{P}\left(\frac{W\left(n h_{n} .\right)}{\left(2 n h_{n} \log N_{n}\right)^{1 / 2}} \in N_{\varepsilon / 2}(f)\right) \\
& -\mathbb{P}\left(\sup _{0 \leq s \leq 1} \frac{\left|\Pi\left(n h_{n} s\right)-n h_{n} s-W\left(n h_{n} s\right)\right|}{\left(2 n h_{n} \log N_{n}\right)^{1 / 2}} \geq \frac{\varepsilon}{2}\right) .
\end{aligned}
$$


Set

$$
\begin{aligned}
P_{n}^{(2)}(f, \varepsilon) & :=\mathbb{P}\left(\frac{W\left(n h_{n} .\right)}{\left(2 n h_{n} \log N_{n}\right)^{1 / 2}} \in N_{\varepsilon / 2}(f)\right) \\
& =\mathbb{P}\left(\frac{W\left(\log N_{n} \cdot\right)}{2^{1 / 2} \log N_{n}} \in N_{\varepsilon / 2}(f)\right) .
\end{aligned}
$$

Note that $N_{\varepsilon / 2}(f)$ is an open set of $B[0,1]$ [endowed with the sup-norm topology]. Therefore, Fact 3 and (2.8) imply for any $n$ sufficiently large

$$
P_{n}^{(2)}(f, \varepsilon) \geq \exp \left(-(1-\delta) \log N_{n}\right),
$$

where $\delta \in(0,1)$ verifies $J\left(N_{\varepsilon / 2}(f)\right)<1-\delta<1$. Now, let us define

$$
P_{n}^{(3)}:=\mathbb{P}\left(\sup _{0 \leq s \leq 1} \frac{\left|\Pi\left(n h_{n} s\right)-n h_{n} s-W\left(n h_{n} s\right)\right|}{\left(2 n h_{n} \log N_{n}\right)^{1 / 2}} \geq \frac{\varepsilon}{2}\right) .
$$

By Fact 2 and (H1)-(H3), we get for any $n$ large enough

$$
\begin{aligned}
P_{n}^{(3)} & \leq \mathbb{P}\left(\sup _{0 \leq s \leq n h_{n}}|\Pi(s)-s-W(s)| \geq \frac{\varepsilon}{4}\left(2 n h_{n} \log N_{n}\right)^{1 / 2}+C_{1} \log \left(n h_{n}\right)\right) \\
& \leq C_{2} \exp \left(-C_{3} \frac{\varepsilon}{4}\left(2 n h_{n} \log N_{n}\right)^{1 / 2}\right) \leq \frac{1}{2} \exp \left(-(1-\delta) \log N_{n}\right) .
\end{aligned}
$$

We jointly use (2.37), (2.39) and (2.41) to obtain

$$
\left(1-P_{n}^{(1)}(f, \varepsilon)\right)^{N_{n}} \leq \exp \left(-\frac{1}{2} \frac{N_{n}^{\delta}}{(\log n)^{1-\delta}}\right) .
$$

By (H3), for any integer $r>1$ and any $n$ large enough, we have

$$
-\frac{N_{n}^{\delta}}{(\log n)^{1-\delta}}<-(\log n)^{r} .
$$

Therefore, for any $n$ sufficiently large

$$
\left(1-P_{n}^{(1)}(f, \varepsilon)\right)^{N_{n}} \leq \exp \left(-\frac{1}{2}(\log n)^{r}\right) .
$$

This last statement implies $\sum_{n} P_{n}(f, \varepsilon)<\infty$. Hence, the Borel-Cantelli lemma entails (2.34).

2.2.3. Proof of Theorem 1.1 (i). Second step. As a second step we prove that, with probability 1

$$
\lim _{n \rightarrow \infty} \delta\left(\left\{d_{n, N}^{-1} \xi_{n}\left(h_{n}, t ; .\right): t \in T_{n}\right\}, \mathcal{S}\right)=0 .
$$

The successive use of Lemma 2.5, the Borel-Cantelli lemma, and Lemmas 2.6 and 2.7 show that (2.45) is true as soon as we have, for any $\varepsilon>0$ and $\gamma>0$

$$
\sum_{k} \mathbb{P}\left(D_{k}(\varepsilon, \gamma)\right)<\infty
$$

For any $k \geq 1$, observe that

$$
\begin{aligned}
& \mathbb{P}\left(d_{n_{k+1}, N_{n_{k+1}}}^{-1} \xi_{n_{k+1}}\left(h_{n_{k+1}}, t ; .\right) \notin \mathcal{S}^{\varepsilon} \text { for a } t \in T\right) \\
& \quad \leq N_{n_{k+1}} \mathbb{P}\left(d_{n_{k+1}, N_{n_{k+1}}}^{-1} \xi_{n_{k+1}}\left(h_{n_{k+1}}, 0 ; .\right) \notin \mathcal{S}^{\varepsilon}\right) .
\end{aligned}
$$

Set

$$
Q_{k}^{(0)}:=\mathbb{P}\left(d_{n_{k+1}, N_{n_{k+1}}}^{-1} \xi_{n_{k+1}}\left(h_{n_{k+1}}, 0 ; .\right) \notin \mathcal{S}^{\varepsilon}\right) .
$$


We use Fact 1 to obtain for any $k \geq 1$,

$$
\begin{aligned}
Q_{k}^{(0)} & \leq 2 \mathbb{P}\left(d_{n_{k+1}, N_{n_{k+1}}}^{-1} L_{n_{k+1}}\left(h_{n_{k+1}}, 0 ; .\right) \notin \mathcal{S}^{\varepsilon}\right) \\
& \leq 2 \mathbb{P}\left(\frac{W\left(n_{k+1} h_{n_{k+1}} .\right)}{\left(2 n_{k+1} h_{n_{k+1}} \log N_{n_{k+1}}\right)^{1 / 2}} \notin \mathcal{S}^{\varepsilon / 2}\right) \\
& +2 \mathbb{P}\left(\sup _{0 \leq s \leq 1} \frac{\left|\Pi\left(n_{k+1} h_{n_{k+1}} s\right)-n_{k+1} h_{n_{k+1}} s-W\left(n_{k+1} h_{n_{k+1}} s\right)\right|}{\left(2 n_{k+1} h_{n_{k+1}} \log N_{n_{k+1}}\right)^{1 / 2}} \geq \frac{\varepsilon}{2}\right) .
\end{aligned}
$$

Now consider

$$
Q_{k}^{(1)}:=\mathbb{P}\left(\frac{W\left(n_{k+1} h_{n_{k+1}} .\right)}{\left(2 n_{k+1} h_{n_{k+1}} \log N_{n_{k+1}}\right)^{1 / 2}} \in B[0,1] \backslash \mathcal{S}^{\varepsilon / 2}\right) .
$$

Observe that $B[0,1] \backslash \mathcal{S}^{\varepsilon / 2}$ is a closed subset of $B[0,1]$ [endowed with the sup-norm topology]. Therefore, Fact 3 and (2.7) entail for any $k$ large enough

$$
Q_{k}^{(1)} \leq \exp \left(-(1+\delta) \log N_{n_{k+1}}\right),
$$

where $\delta>0$ is such that $1<1+\delta<J\left(B[0,1] \backslash \mathcal{S}^{\varepsilon / 2}\right)$. Let

$$
Q_{k}^{(2)}:=\mathbb{P}\left(\sup _{0 \leq s \leq 1} \frac{\left|\Pi\left(n_{k+1} h_{n_{k+1}} s\right)-n_{k+1} h_{n_{k+1}} s-W\left(n_{k+1} h_{n_{k+1}} s\right)\right|}{\left(2 n_{k+1} h_{n_{k+1}} \log N_{n_{k+1}}\right)^{1 / 2}} \geq \frac{\varepsilon}{2}\right) .
$$

By (H1)-(H2) and Fact 2, we have for any $k$ large enough,

$$
\begin{aligned}
Q_{k}^{(2)} & \leq C_{2} \exp \left(-C_{3} \frac{\varepsilon}{2}\left(2 n_{k+1} h_{n_{k+1}} \log N_{n_{k+1}}\right)^{1 / 2}\right) \\
& \leq \exp \left(-(1+\delta) \log N_{n_{k+1}}\right) .
\end{aligned}
$$

Then (H3) and statements (2.21), (2.48), (2.49), (2.51), (2.53) jointly imply

$$
\begin{aligned}
N_{n_{k+1}} Q_{k}^{(0)} & \leq 4 \frac{1}{N_{n_{k+1}}^{\delta}\left(\log n_{k+1}\right)^{1+\delta}} \\
& \leq \frac{2^{1+2 \delta}}{(\log (1+\gamma))^{1+2 \delta}} \frac{1}{(k+1)^{1+2 \delta}} .
\end{aligned}
$$

Hence, we have $\sum_{k} N_{n_{k+1}} Q_{k}^{(0)}<\infty$. Now, use Lemma 2.5, the Borel-Cantelli lemma and Lemmas 2.6 and 2.7 to obtain (2.45).

By (2.34) and (2.45) we conclude that (1.4) is verified. This achieves the proof of Theorem 1.1 (i).

2.3. Proof of Theorem 1.1 (ii). The proof of (1.5) is derived from Theorem A of Deheuvels and Mason [6] since (1.2) holds for any subset $T \subseteq\left[0,1-h_{n}\right]$, finite or infinite. Therefore a detailed proof of (1.5) is omitted.

Remark 2.3. Using the techniques of paragraph 2.2, with a particular set $T_{n}$ of equidistant points, we prove that the sequence of norming constants $c_{n, N}$ is optimal.

\section{Applications}

In this section, we establish new laws of iterated logarithm for kernel density estimators.

Let $\left\{X_{n}: n \geq 1\right\}$ denote a sequence of independent and identically distributed random variables defined on $(\Omega, \mathcal{A}, \mathbb{P})$ with distribution function $G$. We suppose that $G$ is a continuous function on $[A, B],-\infty \leq A<B \leq \infty$, with derivative 
$g(x)=G^{\prime}(x)$, positive and continuous on $[A, B]$. Let $C$ and $D$ denote two constants such that $A<C<D<B$.

Assume $N \geq 1$ and consider the points $x_{1}, \ldots, x_{N}$ of $[A, B]$ such that

$$
A<C<x_{1}=x_{1, N}=x_{1, N_{n}}<\ldots<x_{N}=x_{N, N}=x_{N_{n}, N_{n}}<D<B .
$$

Set $R=R_{N}=R_{N_{n}}=\left\{x_{1}, \ldots, x_{N}\right\}$ and let $K$ be a function satisfying the following assumptions,

(K1) $K$ is of bounded variation on $(-\infty, \infty)$,

(K2) For $M \in(0, \infty), K(u)=0$ for any $|u| \geq M$,

(K3) $\int_{-\infty}^{\infty} K(u) d u=1$.

We introduce the kernel density estimator of Parzen [18] and Rosenblatt [19] (see also Bosq and Lecoutre [1]), defined for any $x \in[A, B]$ by

$$
f_{n}(x)=\left(n h_{n}\right)^{-1} \sum_{i=1}^{n} K\left(\frac{x-X_{i}}{h_{n}}\right),
$$

where $\left\{h_{n}: n \geq 1\right\}$ and $\left\{N_{n}: n \geq 1\right\}$ follow the assumptions (H1)-(H4).

Remark 3.1. Properties of $f_{n}$ can be found in Bosq and Lecoutre [1. See also Stute [24, Deheuvels and Mason [6] among others, for the study of limit laws of the iterated logarithm for the kernel density estimator.

Theorem 3.1. (i) Under (H1), (H2), (H3), (H4) with $d \in[0,1)$ and (K1)-(K3), with probability 1 ,

$$
\begin{aligned}
& \limsup _{n \rightarrow \infty}\left(\frac{n h_{n}}{2 \log N_{n}}\right)^{1 / 2} \max _{x \in\left\{x_{1}, \ldots, x_{N_{n}}\right\}} \pm\left(\frac{f_{n}(x)-\mathbb{E} f_{n}(x)}{\sqrt{g(x)}}\right) \\
& \quad=\left(\int_{-\infty}^{\infty} K^{2}(u) d u\right)^{1 / 2} .
\end{aligned}
$$

(ii) Under (H1), (H2), (H3), (H4) with $d \in[1, \infty]$ and (K1)-(K3), with probability 1 ,

$$
\begin{aligned}
& \lim _{n \rightarrow \infty}\left(\frac{n h_{n}}{2 \log \left(1 / h_{n}\right)}\right)^{1 / 2} \max _{x \in\left\{x_{1}, \ldots, x_{N_{n}}\right\}} \pm\left(\frac{f_{n}(x)-\mathbb{E} f_{n}(x)}{\sqrt{g(x)}}\right) \\
& \quad=\left(\int_{-\infty}^{\infty} K^{2}(u) d u\right)^{1 / 2} .
\end{aligned}
$$

Remark 3.2. Theorem 3.1 may be purposeful for experimental situations where we recall that $f_{n}(x)$ is unknown continuously over $[A, B]$, but only on a discrete subset of $[A, B]$. We can also justify the use of Theorem 3.1 when prebinning methods are considered (see e.g. Härdle [12]).

Proof of Theorem 3.1. Assume that $M=1 / 2$ and let $\widetilde{K}(u):=K(1 / 2-u)$ for $u \in[0,1]$. Next, consider

$$
\widetilde{f}_{n}(x)=\left(n h_{n}\right)^{-1} \sum_{i=1}^{n} \widetilde{K}\left(\frac{X_{i}-x}{h_{n}}\right),
$$

and observe that

$$
\widetilde{f}_{n}(x)=f_{n}\left(x+\frac{1}{2} h_{n}\right)
$$


Proof of (i). The use of (H1) and (K2), combined with the continuity of $g$ on $[A, B]$ implies that (3.2) is true whenever we prove with probability 1 ,

$$
\begin{gathered}
\limsup _{n \rightarrow \infty}\left(\frac{n h_{n}}{2 \log N_{n}}\right)^{1 / 2} \max _{x \in\left\{x_{1}, \ldots, x_{N_{n}}\right\}} \pm\left(\frac{\widetilde{f}_{n}(x)-\mathbb{E} \tilde{f}_{n}(x)}{\sqrt{g(x)}}\right) \\
=\left(\int_{0}^{1} \widetilde{K}^{2}(u) d u\right)^{1 / 2} .
\end{gathered}
$$

For any $x \in R,(3.4)$ entails

$$
\begin{aligned}
\widetilde{f}_{n}(x) & =\int_{0}^{1} h_{n}^{-1} \widetilde{K}(u) d G_{n}\left(x+h_{n} u\right) \\
& =-\int_{0}^{1} h_{n}^{-1}\left(G_{n}\left(x+h_{n} u\right)-G_{n}(x)\right) d \widetilde{K}(u),
\end{aligned}
$$

where $G_{n}$ is the empirical distribution function based on $X_{1}, \ldots, X_{n}$. Next using the equality $G_{n}(x)=F_{n}(G(x))$ (see e.g. Shorack and Wellner [21 pp. 3-5]), we see that

$$
n^{1 / 2} h_{n}\left(\widetilde{f}_{n}(x)-\mathbb{E} \widetilde{f}_{n}(x)\right)=-\int_{0}^{1}\left(\alpha_{n}\left(G\left(x+h_{n} u\right)\right)-\alpha_{n}(G(x))\right) d \widetilde{K}(u) .
$$

Let $\delta_{0}>0$ be a constant whose definition will be given elsewhere. Consider the subset $\widetilde{R}=\widetilde{R}_{N}=\widetilde{R}_{N_{n}}=\left\{\widetilde{x}_{1}, \ldots, \widetilde{x}_{k_{N_{n}}}\right\} \subseteq R$, such that for any $n$ sufficiently large we have $\widetilde{x}_{k_{N_{n}}}-\widetilde{x}_{1}<\delta_{0}$.

Next, we define the dual set of $\widetilde{R}, \widetilde{T}=\widetilde{T}_{N}=\widetilde{T}_{N_{n}}=\left\{\widetilde{t}_{1}, \ldots, \widetilde{t}_{k_{N_{n}}}\right\} \subseteq T^{\prime}$, with $G\left(\widetilde{x}_{1}\right)=\widetilde{t}_{1}<\ldots<G\left(\widetilde{x}_{k_{N_{n}}}\right)=\widetilde{t}_{k_{N_{n}}}$. For any $n \geq 1$, define the sequence of constants $a_{n}=\Lambda h_{n}$, where $\Lambda>0$ will be described elsewhere. It is easy to see that

$$
\begin{aligned}
& \left\{\left(2 a_{n} \log \left(N_{n} / \Lambda\right)\right)^{-1 / 2} \xi_{n}\left(a_{n}, t ; .\right): t \in \widetilde{T}\right\} \\
& \quad=\left\{\left(2 a_{n} \log \left(N_{n} / \Lambda\right)\right)^{-1 / 2}\left(\alpha_{n}\left(G(x)+\Lambda h_{n} .\right)-\alpha_{n}(G(x))\right): x \in \widetilde{R}\right\} .
\end{aligned}
$$

Moreover $\left\{a_{n}: n \geq 1\right\}$ verifies assumptions (H1), (H2), (H3), (H4) with $d \in[0,1)$. Hence, for $\varepsilon>0$, statement (1.4) implies with probability 1 for any $n$ sufficiently large,

$$
\left\{\left(2 a_{n} \log \left(N_{n} / \Lambda\right)\right)^{-1 / 2} \xi_{n}\left(a_{n}, t ; .\right): t \in \widetilde{T}\right\} \subseteq \mathcal{S}^{\varepsilon / 8} .
$$

Next, we set $\Lambda=\max _{1 \leq i \leq k_{N}} g\left(x_{i}\right)$. For any $x \in \widetilde{R}, u \in[0,1]$, define

$$
\Theta_{n}(x ; u):=\frac{G\left(x+h_{n} u\right)-G(x)}{\Lambda h_{n}} .
$$

Recall that $g$ is continuous on $[A, B]$. Hence, we choose $\delta_{0}>0$ such that for any $n$ large enough and $x \in \widetilde{R}$,

$$
\left|\Lambda^{-1} g(x)-1\right|<\frac{\varepsilon^{2}}{32} .
$$

Moreover, the continuity of $G,(3.10)$ and (3.11) jointly imply for any $n$ large enough, $u \in[0,1]$ and $x \in \widetilde{R}$,

$$
\left|\Theta_{n}(x ; u)-u\right| \leq \frac{\varepsilon^{2}}{16}
$$


For any $t \in \widetilde{T}$ and $u \in[0,1]$, define $\Xi(u):=\left(2 a_{n} \log \left(N_{n} / \Lambda\right)\right)^{-1 / 2} \xi_{n}\left(a_{n}, t ; u\right)$ and observe that for any $f \in B[0,1]$,

$$
\begin{aligned}
& \left(2 a_{n} \log \left(N_{n} / \Lambda\right)\right)^{-1 / 2}\left(\alpha_{n}\left(G\left(x+h_{n} .\right)\right)-\alpha_{n}(G(x))\right)-f \| \\
& \quad \leq\left\|\Xi\left(\Theta_{n}(x ; .)\right)-\Xi(.)\right\| \\
& \quad+\left\|\left(2 a_{n} \log \left(N_{n} / \Lambda\right)\right)^{-1 / 2}\left(\alpha_{n}\left(G(x)+\Lambda h_{n} .\right)-\alpha_{n}(G(x))\right)-f\right\| .
\end{aligned}
$$

By (3.9), observe that $\Xi \in \mathcal{S}^{\varepsilon / 8}$. Therefore, a simple computation prove that $\left\|\Xi\left(\Theta_{n}(x ;).\right)-\Xi().\right\| \leq \varepsilon / 2$. Moreover for any $f \in \mathcal{S},(3.9)$ implies

$$
\left\|\left(2 a_{n} \log \left(N_{n} / \Lambda\right)\right)^{-1 / 2}\left(\alpha_{n}\left(G(x)+\Lambda h_{n} .\right)-\alpha_{n}(G(x))\right)-f\right\| \leq \frac{\varepsilon}{8}
$$

Hence, (3.13) entails with probability 1 for any $n$ large enough

$$
\left\{\left(2 a_{n} \log \left(N_{n} / \Lambda\right)\right)^{-1 / 2}\left(\alpha_{n}\left(G\left(x+h_{n} .\right)\right)-\alpha_{n}(G(x))\right): x \in \widetilde{R}\right\} \subseteq \mathcal{S}^{5 \varepsilon / 8} .
$$

Next we use (3.7) and (3.14) to obtain for any $n$ sufficiently large

$$
\begin{gathered}
\frac{n^{1 / 2} h_{n}}{\left(2 a_{n} \log \left(N_{n} / \Lambda\right)\right)^{1 / 2}} \max _{x \in \widetilde{R}} \pm\left(\widetilde{f}_{n}(x)-\mathbb{E} \widetilde{f}_{n}(x)\right) \\
\leq \frac{5 \varepsilon}{8} \int_{0}^{1}|d \widetilde{K}(u)|+\sup _{f \in \mathcal{S}} \mp \int_{0}^{1} f(u) d \widetilde{K}(u) .
\end{gathered}
$$

We use (K2), (K3), and Schwarz's inequality to obtain

$$
\left(\int_{0}^{1} f(u) d \widetilde{K}(u)\right)^{2} \leq\left(\int_{0}^{1} \widetilde{K}^{2}(u) d u\right) .
$$

Moreover, for any $\varepsilon^{\prime}>0$ sufficiently small, (3.11) entails for any $n$ large enough

$$
\left(\frac{\Lambda}{g(x)}\right)^{1 / 2}\left(1+\frac{\log (1 / \Lambda)}{\log N_{n}}\right)^{1 / 2} \leq\left(1-\frac{\varepsilon^{2}}{32}\right)^{-1}(1+\varepsilon)^{1 / 2} \leq 1+\varepsilon^{\prime}
$$

Therefore, (2.69)-(2.71) imply almost surely for any $n$ large enough,

$$
\begin{aligned}
& \left(\frac{n h_{n}}{2 \log N_{n}}\right)^{1 / 2} \max _{x \in \widetilde{R}} \pm\left(\frac{\widetilde{f}_{n}(x)-\mathbb{E} \widetilde{f}_{n}(x)}{\sqrt{g(x)}}\right) \leq \frac{5 \varepsilon}{8}\left(1+\varepsilon^{\prime}\right) \\
& \quad \times \int_{0}^{1}|d \widetilde{K}(u)|+\left(1+\varepsilon^{\prime}\right)\left(\int_{0}^{1} \widetilde{K}^{2}(u) d u\right)^{1 / 2}+\varepsilon .
\end{aligned}
$$

For $\delta_{0}>0$ sufficiently small, $R$ can be seen as a finite union of sets $\widetilde{R}_{i}$ [similar to $\widetilde{R}$ ] with $1 \leq i \leq L<\infty$. Therefore, using the same proof as the one developed earlier, inequality (3.18) can be obtained for each $\widetilde{R}_{i}, 1 \leq i \leq L$, and as a consequence, for any $x \in R$. Next, we let $\varepsilon$ and $\varepsilon^{\prime}$ converge to 0 to obtain (3.2).

Proof of (ii). The proof of (3.3) is very similar to the one developed before and therefore omitted.

\section{REFERENCES}

[1] Bosq D. and Lecoutre J.P. (1987). Théorie de l'estimation fonctionnelle. Economie et Statistiques avancées. Economica, Paris.

[2] Chernoff H. (1952). A measure of asymptotic efficiency for tests of a hypothesis based on the sum of observations. Annals of Mathematical Statistics, 23, 493-507. MR 15:241e

[3] Conway J.B. (1985). A course in functional analysis. Springer, Berlin. MR 86h:46001 
[4] Deheuvels P. (1992). Functional laws of the iterated logarithm for large increments of empirical and quantile processes. Stochastic Processes and their Applications, 43, 133-163. MR 93m:60057

[5] Deheuvels P. and Mason D.M. (1990). Nonstandard functional laws of the iterated logarithm for tail empirical and quantile processes. Annals of Probability, 18, 1693-1722. MR 91j:60056

[6] Deheuvels P. and Mason D.M. (1992). Functional laws of the iterated logarithm for the increments of empirical and quantile processes. Annals of Probability, 20, 1248-1287. MR 93h:60046

[7] Deheuvels P. and Mason D.M. (1994). Random fractals generated by oscillations of processes with stationary and independent increments. Probability in Banach spaces, Sandjberg, 9, 73-89. MR 96b:60191.

[8] Deheuvels P. and Mason D.M. (1995). On the fractal nature of empirical increments. Annals of Probability, 23, 355-387. MR 96e:60064

[9] Deheuvels P. and Révész P. (1986). Weak laws for the increments of Wiener processes, Brownian bridges, empirical processes and partial sums of I.I.D.R.V.'s. Mathematical Statistics and Probability theory, Vol. A, Bad Tatzmannsdorf, 69-88. MR 89a:60057

[10] Deuschel J.D. and Stroock D.W. (1989). Large Deviations. Academic Press, New York. MR 90h:62006

[11] Dindar Z. (1998). Some more results on increments of the partially observed empirical process. Submitted

[12] Härdle W. (1991). Smoothing techniques. With implementation in S. Springer, New York. MR 93b:62006

[13] James B.R. (1975). A functional law of the iterated logarithm for weighted empirical distributions. Annals of Probability, 3, 762-772. MR 53:6695

[14] Komlós J., Major P. and Tusnády G. (1975a). Weak convergence and embedding. Colloquia Mathematica Societatis János Bolyai, North-Holland, Amsterdam, 11, 149-165. MR 53:6676

[15] Komlós J., Major P. and Tusnády G. (1975b). An approximation of partial sums of independent r.v.'s and the sample d.f. I. Z. Wahrsch. Verw. Gebiete, 32, 111-131. MR 51:11605b

[16] Komlós J., Major P. and Tusnády G. (1976). An approximation of partial sums of independant r.v.'s and the sample d.f. II. Z. Wahrsch. Verw. Gebiete, 34, 33-58. MR 53:6697

[17] Lifshits M.A. and Weber M. (1997). Strassen laws of iterated logarithm for partially observed processes. Journal of Theoretical Probability, 10, 101-115. MR 98h:60041

[18] Parzen E. (1962). On estimation of a probability density function and mode. Annals of Mathematical Statistics, 33, 1065-1076. MR 26:841

[19] Rosenblatt M. (1979). Global measures of deviation for kernel and nearest neighbor density estimates. Smoothing techniques for curve estimation, Lecture Notes in Mathematics, 757. Springer, Berlin. MR 81g:62076

[20] Schilder M. (1966). Some asymptotic formulae for Wiener integrals. Transactions of the American Mathematical Society, 125, 63-85. MR 34:1770

[21] Shorack G.R. and Wellner J.A. (1986). Empirical processes with applications to statistics. Wiley, New York. MR 88e:60002

[22] Strassen V. (1964). An invariance principle for the law of the iterated logarithm. Z. Wahrsch. Verw. Gebiete, 3, 211-226. MR 30:5379

[23] Stute W. (1982a). The oscillation behaviour of empirical process. Annals of Probability, 10, 86-107. MR 83e:60028

[24] Stute W. (1982b). A law of the iterated logarithm for kernel density estimators. Annals of Probability, 10, 414-422. MR 84e:62060

[25] Varadhan S.R.S. (1966). Asymptotic probabilities and differential equations. Communications on Pure and Applied Mathematics, 19, 261-286. MR 34:3083

[26] Weber M. (1988). The law of the iterated logarithm for subsequences in Banach spaces. Probability in Banach spaces, 7, 269-285. MR 92h:60009

[27] Weber M. (1990). The law of the iterated logarithm on subsequences-characterizations. Nagoya Mathematical Journal, 118, 65-97. MR 92a:60089

L.S.T.A., Université Paris VI, 45 rue Vineuse, 75016 Paris, France

E-mail address: dindar@ccr.jussieu.fr 